\title{
Untypical autoimmune pancreatitis and pancreatic cancer: differential diagnosis experiences extracted from misdiagnose of two cases
}

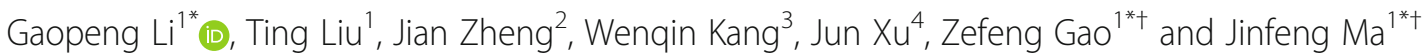

\begin{abstract}
Background: Differentiation between pancreatic cancer (PC) and focal form of autoimmune pancreatitis (AIP) is very challenging, with similar clinical presentations, laboratory results and morphologic imagings of US, CT, EUS, MRI, ERCP, PET-CT. Even serum IgG4 and biopsy sometimes cannot give clear-cut differential accurate diagnostis. Considering the totally different management strategy of the two diseases, accurate diagnostic value is urgently needed to remind the clinicians of the rare diagnosis of untypical AIP among frequent PC-suspected patients.

Results: We present 2 laparotomy cases of AIP that had a high similar characteristic to PC and retrospectively extracted the warning signs that may help select untypical AIP in PC-suspected patients.

Conclusions: We find that mild fluctuating jaundice with abdominal pain, young age, tumor marker of TPS, TPA and diverse results between variable radiological tests can help to differentiate AIP mass from PC, through retrospectively analyzing work-up process of AIP in two patients who underwent laparotomy for suspected PC.
\end{abstract}

Keywords: IgG4, Biopsy, Pancreatic cancer, Autoimmune pancreatitis

\section{Background}

Autoimmune pancreatitis (AIP) is a unique pancreatic manifestation of systemic immunoglobulin G4 (IgG4)-related sclerosing disease, histopathologically characterized by abundant infiltration of IgG4-positive lymphoplasmacyte and fibrosis of the pancreas with obliterative phlebitis [1]. However, contrary to typical AIPs, patients with untypical local lesion AIP and pancreatic cancer (PC) share similar clinical presentations, laboratory measurements, morphologic features of radiological examinations. To date, measurement of serum IgG4 has become a useful tool for their differentiation. However, several studies report pancreatic masses in patients with 1.6 times the upper limit of

\footnotetext{
* Correspondence: malone2001@163.com; 18434302162@163.com; mjinfeng99@163.com

† Jinfeng Ma and Zefeng Gao contributed equally to this work and should be considered co-corresponding authors

${ }^{1}$ Department of general surgery, Shanxi Cancer Hospital, Shanxi Medical

University, Taiyuan, Shanxi Province, China

Full list of author information is available at the end of the article
}

normal serum IgG4 levels (> $135 \mathrm{mg} / \mathrm{dL}$ ) histopathologically proven to be PC. Moreover, quantification of serum IgG4 is often variable and inaccurate due to lack of standardization in IgG subclass assay calibration [2]. EUSguided fine needle aspiration (FNA) may be of additional value in histological confirmation. The major limitations of the technique are operator dependence and high rate of false-negative results due to inadequate sample provided. In addition, even core biopsies will not provide enough tissue to distinguish pathological characteristics between AIP and $\mathrm{PC}$, especially in $\mathrm{PC}$ patients with concurrent chronic pancreatitis. Also, clinically, most of PC-suspected patients cannot routinely have measurement of serum IgG4 concentrations or pre-operative histological confirmation to exclude relative rare untypical AIP. In all, as clear-cut diagnostic tool is not readily available for untypical AIP, simple diagnostic tool is urgently needed to guide the clinician in the decision-making process. Here, we present 2 laparotomy cases of AIP that had a high similar characteristic to

(c) The Author(s). 2019 Open Access This article is distributed under the terms of the Creative Commons Attribution 4.0 International License (http://creativecommons.org/licenses/by/4.0/), which permits unrestricted use, distribution, and reproduction in any medium, provided you give appropriate credit to the original author(s) and the source, provide a link to the Creative Commons license, and indicate if changes were made. The Creative Commons Public Domain Dedication waiver (http://creativecommons.org/publicdomain/zero/1.0/) applies to the data made available in this article, unless otherwise stated. 
PC and retrospectively extracted the warning signs for discrimination of untypical AIP in PC-suspected patients.

\section{Methods}

We conducted two laparotomy cases of AIP that had a high similar characteristic to PC in Shanxi cancer hospitals in western China from June from July 2016 to August 2018. Both patients received routine treatment and care of abdominal surgery, according to medical ethics. The subjective feeling and objective data were all documented in case history. All the treatments and tests obtained informed consent of both patients.

\section{Results}

\section{Case 1}

A 34-year-old female visited our hospital in July 2016 because of a 4-month history of intermittent epigastralgia and poor appetite. The symptoms were not associated with food intake or daily exercise, and there was no relieving or aggravating factor. After admission, body check showed no swelling of the salivary glands and the cervical lymph nodes were not palpable. Her laboratory tests revealed elevated liver enzymes, including glutamate pyruvate transaminase (ALT) of $449 \mathrm{IU} / \mathrm{L}$ (reference range, 9-60 IU/L), glutamate oxaloacetate transaminase (AST) of $383 \mathrm{IU} / \mathrm{L}$ (reference range, 15-45 IU/L), gamma Glutamyl transpeptidase (GGT) of $823 \mathrm{IU} / \mathrm{L}$ (reference range, 10-60 IU/L, alkaline hosphate (ALP) of $1170 \mathrm{IU} / \mathrm{L}$ (reference range, 35-100 IU/L), total bilirubin (Tbil) of $183 \mu \mathrm{mol} / \mathrm{L}$ (reference range, $1.7-21 \mathrm{mg} / \mathrm{dL}$ ), conjugated bilirubin (Dbil) of $142.1 \mu \mathrm{mol} / \mathrm{L}$ (reference range, 0.0$6.8 \mu \mathrm{mol} / \mathrm{L}$ ), and unconjugated bilirubin (Ibil) of $40.9 \mu \mathrm{mol} /$ $\mathrm{L}$ (reference range, $1.7-14.2 \mu \mathrm{mol} / \mathrm{L}$ ). The results of other laboratory tests, including cholesterol profile, electrolytes, a complete blood count/differential count, renal function parameters and most tumor markers were within the normal range except high elevation of CA50, CA19-9, CA242, TPS and TPA (Table 1). Esophagogastroduodenoscopy revealed enlargement of duodenal papilla and external compression of the duodenum, which raised suspicion for a pancreatic tumor. Abdominal ultrasonography showed a mass in the uncinate process of the pancreas. Abdominal computed tomography (CT) with contrast enhancement revealed a mass arising in the end of dilated lower bile duct. Abdominal MRI depicted dilatation of the intrahepatic, extrahepatic bile ducts and main pancreatic duct caused by $5.5-\mathrm{cm}$ mass lesion in the pancreatic head, with encasement of superior mesenteric vein. 18F-fluorodeoxyglucose (FDG) positron emission tomography (PET)/CT was performed and showed an FDG-avid, hypermetabolic, swollen soft tissue mass in the pancreatic head with a maximum standardized uptake value of 8.3. Adjacent low-grade FDG-avid lymph nodes with a maximum standardized uptake value of 3.0 were also noted. No FDG-avid lesions were present in the bilateral salivary glands, retroperitoneal spaces, orbiliary tracts [3]. In all, these findings were highly suggestive of obstructive jaundice due to a malignant pancreatic tumor with no distant metastasis. As the patient refuse to take US-guided biopsy and PC was highly suspected, the patient underwent pancreaticoduodenectomy and recover well. However, postoperative histologic analysis of the pancreatic head revealed moderate lymphoplasmacytic infiltration with obliterative venulitis and stromal fibrosis. Immunohistochemically, abundant IgG4-positive plasma cells $(>20 / \mathrm{hpf}$ and $\operatorname{IgG} 4+/ \mathrm{IgG}+$ plasma cell ratio $>40 \%)$ were observed infiltrating the head of the pancreas, consistent with AIP [3]. Further examination showed all the serum immune antibody including IgG4 were within normal range except slightly elevation of IgM and AMA (Table 2).

Table 1 Changes of tumor markers in two cases

\begin{tabular}{|c|c|c|c|c|c|c|c|}
\hline \multirow[b]{2}{*}{ time } & \multicolumn{3}{|l|}{ Case 1} & \multicolumn{3}{|l|}{ Case 2} & \multirow[t]{2}{*}{ Reference } \\
\hline & 27 days & 31 days & 37 days & 3 days & 15 days & 64 days & \\
\hline CEA & 1.46 & 0.84 & 0.75 & 1.5 & 0.80 & 0.15 & $<3 \mu \mathrm{g} / \mathrm{L}$ \\
\hline CA199 & $426.50 \uparrow$ & $227.89 \uparrow$ & $282.22 \uparrow$ & 1.1 & 2.05 & 0.55 & $<20 \mathrm{U} / \mathrm{mL}$ \\
\hline CA242 & & $43.34 \uparrow$ & $40.92 \uparrow$ & & 1.03 & 1.65 & $<12 \mathrm{U} / \mathrm{mL}$ \\
\hline AFP & 2.92 & 4.35 & 4.16 & 6.0 & 5.17 & 6.15 & $<15 \mu \mathrm{g} / \mathrm{L}$ \\
\hline CA724 & & 4.25 & 1.55 & & 0.82 & 0.66 & $<10 \mathrm{U} / \mathrm{mL}$ \\
\hline CA50 & $222.70 \uparrow$ & 32.44 & 51.52 & & & 13.55 & $<20 \mathrm{U} / \mathrm{mL}$ \\
\hline SCC & & 0.24 & 0.27 & & & 0.19 & $<1 \mathrm{ng} / \mathrm{mL}$ \\
\hline TPA & & $5.25 \uparrow$ & $9.28 \uparrow$ & & & $14.63 \uparrow$ & $<2 \mathrm{ng} / \mathrm{mL}$ \\
\hline TPS & & $234.57 \uparrow$ & $765.82 \uparrow$ & & & $1010.07 \uparrow$ & $<150 \mathrm{U} / \mathrm{L}$ \\
\hline VEGF & & 300.00 & 257.66 & & & 617.26 & $62-707 \mathrm{pg} / \mathrm{mL}$ \\
\hline CA125 & 28 & & & & & 5.75 & $<35 \mathrm{U} / \mathrm{mL}$ \\
\hline CA153 & 7.5 & & & & & & $<31.3 \mathrm{U} / \mathrm{mL}$ \\
\hline
\end{tabular}


Table 2 Serological immune realted antibody: $\uparrow$ represent higher than reference, $\downarrow$ represent lower than reference. Immunoturbidimetry (ITM), Western blotting (WB), indirect immunofluorescence (IFL), blank no test

\begin{tabular}{|c|c|c|c|c|c|}
\hline & \multirow{2}{*}{$\begin{array}{l}\text { Case } 1 \\
\text { (postoperative) }\end{array}$} & \multicolumn{2}{|l|}{ Case 2} & \multirow[t]{2}{*}{ Reference (unit) } & \multirow[t]{2}{*}{ method } \\
\hline & & preoperative & postoperative & & \\
\hline $\lg A$ & 1.66 & 2.14 & 2.23 & $0.7-4.0 \mathrm{~g} / \mathrm{L}$ & Elisa \\
\hline $\lg G$ & 13.34 & 11.34 & 16.3 & $7-16 \mathrm{~g} / \mathrm{L}$ & Elisa \\
\hline $\lg M$ & $3.25 \uparrow$ & 0.67 & 0.909 & $0.4-2.3 \mathrm{~g} / \mathrm{L}$ & Elisa \\
\hline $\lg E$ & & & $657.50 \uparrow$ & $20 \sim 200 \mathrm{IU} / \mathrm{mL}$ & Elisa \\
\hline $\mathrm{t}-\mathrm{PSA}$ & & & 0.831 & $0-4 \mathrm{ng} / \mathrm{mL}$ & Elisa \\
\hline f-PSA & & & 0.301 & $0-4 \mathrm{ng} / \mathrm{mL}$ & Elisa \\
\hline ASO & & & $<25.0$ & $0-116 \mathrm{IU} / \mathrm{mL}$ & Elisa \\
\hline Anti-CCP & 1.75 & & & $0-25 \mathrm{IU} / \mathrm{mL}$ & Elisa \\
\hline anti-TB & negative & & & negative & Elisa \\
\hline AMA-M2 & 8.12 & & & $0-25 \mathrm{IU} / \mathrm{mL}$ & Elisa \\
\hline Anti-a-Fodrin & negative & & & negative & Elisa \\
\hline Complement 3 & 1.09 & & $0.740 \downarrow$ & $0.79-1.52 \mathrm{~g} / \mathrm{L}$ & ITM \\
\hline Complement 4 & 0.14 & & $0.069 \downarrow$ & $0.1-0.4 \mathrm{~g} / \mathrm{L}$ & ITM \\
\hline RF & 1.95 & & $73.1 \uparrow$ & $<20 \mathrm{IU} / \mathrm{mL}$ & ITM \\
\hline $\lg G 1$ & & & $1170.0 \uparrow$ & 405-1011 mg/dL & ITM \\
\hline $\lg G 2$ & & & 234 & $169-768 \mathrm{mg} / \mathrm{dL}$ & ITM \\
\hline $\lg G 3$ & & & 32.8 & $11-85 \mathrm{mg} / \mathrm{dL}$ & ITM \\
\hline $\lg G 4$ & 5.43 & 98 & $266.0 \uparrow$ & $<201 \mathrm{mg} / \mathrm{dL}$ & ITM \\
\hline ANA & negative & negative & & negative & $\mathrm{IFL}$ \\
\hline AKA & negative & & & negative & $\mathrm{IFL}$ \\
\hline ASMA & negative & & & negative & $\mathrm{IFL}$ \\
\hline AMA & $1: 100 \uparrow$ & & & negative & $\mathrm{IFL}$ \\
\hline AMA-M2 & negative & negative & & negative & WB \\
\hline Sp100 & negative & negative & & negative & WB \\
\hline LKM1 & negative & negative & & negative & WB \\
\hline Gp210 & negative & negative & & negative & WB \\
\hline LC1 & negative & negative & & negative & WB \\
\hline SLA & negative & negative & & negative & WB \\
\hline Anti-Nucleosomes & negative & & & negative & WB \\
\hline Anti-dsDNA & negative & & & negative & WB \\
\hline SmD1 & negative & & & negative & WB \\
\hline Anti-PO & negative & & & negative & WB \\
\hline Anti-Histones & negative & & & negative & WB \\
\hline U1-SnRNP & negative & & & negative & WB \\
\hline Anti-SSA/Ro60 & negative & & & negative & WB \\
\hline Anti-SSA/Ro52 & negative & & & negative & WB \\
\hline $\mathrm{SSB} / \mathrm{La}$ & negative & & & negative & WB \\
\hline Anti-Slc-70 & negative & & & negative & WB \\
\hline Anti-CENP-B & negative & & & negative & WB \\
\hline Anti-Jo-1 & negative & & & negative & WB \\
\hline
\end{tabular}




\section{Case 2}

A 49-year-old male was admitted to our hospital in August 2018 complaining of epigastralgia and jaundice. He had no history of swollen salivary glands and the cervical lymph nodes were not palpable. Laboratory data included: ALT of $57 \mathrm{IU} / \mathrm{L}$ (reference range, 9-60 IU/L), AST of $42 \mathrm{IU} / \mathrm{L}$ (reference range, 15-45 $\mathrm{IU} / \mathrm{L}$ ), GGT of $133 \mathrm{IU} / \mathrm{L}$ (reference range, 10-60 IU/L, ALP of $191 \mathrm{IU} / \mathrm{L}$ (reference range, 455-125 IU/L), Tbil of $147.4 \mu \mathrm{mol} / \mathrm{L}$ (reference range, $1.7-21 \mathrm{mg} / \mathrm{dL}$ ), Dbil of $1.5 .6 \mu \mathrm{mol} / \mathrm{L}$ (reference range, $0.0-6.8 \mu \mathrm{mol} / \mathrm{L}$ ), and Ibil of $41.8 \mu \mathrm{mol} / \mathrm{L}$ (reference range, $1.7-14.2 \mu \mathrm{mol} / \mathrm{L}$ ). The results of other laboratory tests, including a complete blood count/differential count, cholesterol profile, electrolytes, renal function parameters, serum IgG4 were within the normal range (Table 2). The Changes of tumor markers are summarized in Table 1. Overall, most tumor markers were within the normal range except high elevation of TPS and TPA. Abdominal ultrasonography demonstrated a $4 \mathrm{~cm}$-sized hypoechoic mass in the pancreatic head with dilatation of the intrahepatic and extrahepatic bile ducts and the main pancreatic duct. The mass displayed hyper-enhancement in arterial phase and hypoenhancement in the portal and delayed phase on CEUS, typical "fast-in and fast-out" contrast pattern of maligant tumor. Helical CT and MRI scan with contrast enhancement, revealed a nodule in the end of the dilated lower bile in the enlarged pancreatic head, with dilatation of the intrahepatic and extrahepatic bile ducts and the main pancreatic duct, stenosis or obliteration of the pancreatic portion of the common bile duct. The mass was hypoattenuated to the pancreas in the early phase, but attenuation increased in the delayed phase. Given the diagnosis of obstructive jaundice due to malignant pancreatic tumor, the patients choose palliative operative intervention of hepaticojejunostomy. 1 month after recovering and checking out, the patient was readmitted to our hospital complaining of jaundice recurrence. Laboratory data included: ALT of $498 \mathrm{IU} / \mathrm{L}$ (reference range, 9-60 IU/L), AST of $397 \mathrm{IU} / \mathrm{L}$ (reference range, 15-45 IU/L), GGT of $734 \mathrm{IU} / \mathrm{L}$ (reference range, 10-60 IU/L, ALP of $420 \mathrm{IU} / \mathrm{L}$ (reference range, $455-125 \mathrm{IU} / \mathrm{L}$ ), Tbil of $119.6 \mu \mathrm{mol} / \mathrm{L}$ (reference range, $1.7-21 \mathrm{mg} / \mathrm{dL}$ ), Dbil of $55.8 \mu \mathrm{mol} / \mathrm{L}$ (reference range, $0.0-6.8 \mu \mathrm{mol} / \mathrm{L}$ ), and Ibil of $63.8 \mu \mathrm{mol} / \mathrm{L}$ (reference range, 1.7-14.2 $\mu \mathrm{mol} / \mathrm{L}$ ). MRI displayed an inflammatory infiltrate in the swollen pancreas, dilatation of the intrahepatic bile ducts and thicken edema wall of extrahepatic bile ducts, which was compatible with AIP. Further laboratory tests revealed the serum IgG4 was elevated to $266 \mathrm{mg} / \mathrm{dL}$ this time. The patient is skeptical for AIP and was given corticosteroid treatment as an outpatient and was well 11 months later. Since the operation, his serum IgG4 levels have decreased to be 40.3 (Table 2). Based on the postoperative recurrence of jaundice, elevated serum IgG4 level, MRI findings and good response to corticosteroid treatment, the diagnosis of AIP was then confirmed, although the specimen of bile cyst and regional lymph nodes showed no fibrous tissue with focal sclerotic stroma, focal lymphoid cell aggregation or IgG4-positive plasma cells.

\section{Discussion and conclusions}

Since 2002, several diagnostic criteria for AIP and their amendments has been proposed by Asian countries (Japan and Korea) [4], USA (HISORt, Mayo Clinic) [5] and International Association of Pancreatology (International Consensus diagnostic criteria of Experts from Asia, North America, and Europe) [6]. With subtle inconsistency, clinical AIP diagnosis normally accord following consensus. (1): In the patients with typical AIP, US, CT and MRI were less likely to find a discrete pancreatic mass and more likely to find a diffusely enlarged pathological pancreatic head in a swollen pancreas [7]. ERCP and MRCP generally shows fairly distinctive irregular narrowing of the dilated main pancreatic duct [3]. However, focal form of AIP in the present study and PC share many features, including sudden jaundice, elevation of tumor markers, and imaging manifestations of focal mass in the pancreas, "double-duct sign" representing stricture in both the biliary and pancreatic ducts, and angiographic abnormalities, which make the discrimination very challenging. Also, the positive predictive value of radiological diagnosis for pancreatic cancer declines in patients with concurrent chronic pancreatitis [7]. (2): Typical cases also have markedly elevated serum IgG4 (serological cut-off $>135 \mathrm{mg} / \mathrm{dL}$ ), hypergammaglobulinemia and a favorable response to steroid therapy [8-10]. However, the characteristic increased serum IgG4 level was not observed after operation in the first AIP case, which is confirmed by postoperative histological and immunohistochemical characteristics. In the second case, preoperative serological IgG4 are with normal range $(98 \mathrm{mg} / \mathrm{dL})$, which elevated to $266 \mathrm{mg} / \mathrm{dL}$ after surgery and decreased to $40.6 \mathrm{mg} / \mathrm{dL}$ after 3 months of corticosteroid treatment. Several studies report pancreatic masses in patients with particularly high serum IgG4 levels (> $135 \mathrm{mg} / \mathrm{dL}$ ) histopathologically proven to be PC. Cases of the present study further declines the positive predictive value of elevated serum IgG4 levels (> $135 \mathrm{mg} /$ dL) for AIP. The reason may be serum IgG4 level changes with the immune functional status, encompassing genetic predisposition, intrinsic and extrinsic triggers and their interaction with the immune system. The outcome of these processes lead to dynamic changes of activation of immune cells and cytokine levels, which in turn leads to spontaneously relapsing and remitting inflammatory injury in pancreas [11-13]. (3): Histological confirmation by biopsy and brush cytology, often guided by endoscopic 
ultrasound and ERCP respectively, could aid in making a correct diagnosis when there is uncertainty over the differentiation of PC from AIP. The major limitations of the technique are operator dependence and a limited field of visualization for detecting metastatic spread to the liver and peritoneum. Furthermore, there is a high rate of falsenegative results as seen in a previous study, especially in patients who have the smallest lesions and/or in the presence of chronic pancreatitis, due to the inadequate sample provided (even by core biopsies) [3, 14]. In addition, as an autoimmune disease, the dynamic interaction between endogenous and exogenous factors and the immune system play a key role in the pathogenesis and propagation of inflammation as well as healing process, which may make preoperative histological test miss the histological and immunohistochemical characteristic of AIP. This may explain that the specimen of bile cyst and regional lymph nodes showed no fibrous tissue with focal sclerotic stroma, focal lymphoid cell aggregation or IgG4-positive plasma cells. Also, clinically, histologic diagnosis is not usually required for PC surgery to go ahead and most of PC-suspected patients cannot routinely have pre-operative histological confirmation to exclude relative rare untypical
AIP. (4): Nonoperative management strategy with steroids are frequently used as diagnostic therapy of AIP [15]. However, with risk of delaying PC treatment, they are more suitable for following known AIP cases rather than preoperative differentiation diagnosis from PC. In all, considering the absence of preoperative clear-cut differential diagnostic tool and the fact of up to $10 \%$ of the PCsuspected patients undergoing unnecessary laparotomy for absence of typical characters of AIP [7] (just like both of the two cases here), extracting diagnostic value from routine presenting signs and symptoms is urgently needed to guide the clinician in the decision-making process.

We extracted several characteristics that may help discriminate AIP from PC, through retrospectively analyzing work-up process of AIP in two patients who underwent laparotomy for suspected PC. (1): First, we found surprisingly that Liver function tests of both the two patients showed combination of results indicative of intermittent cholestasis and liver damage (mild raised but fluctuating levels of liver enzymes including Tbil, Dbil, Ibil, AST, ALT, ALP and GGT (Fig. 1). That intermittent jaundice which disappears spontaneously is obvious different to the progressive jaundice of PC and

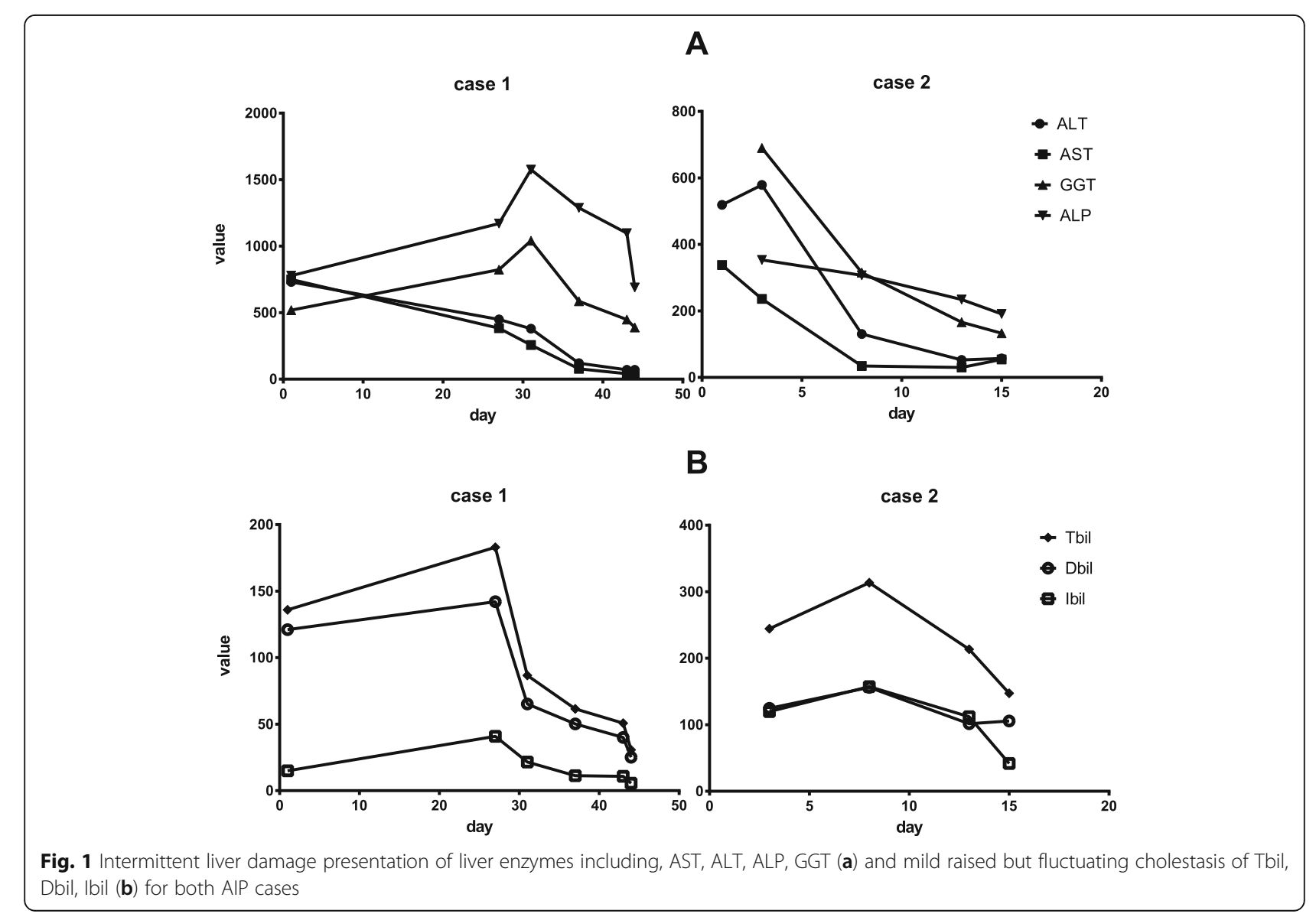


periampullary carcinoma, which represent highly elevated liver enzymes during short time [16, 17]. Contrary to the Hopkins and Mayo experience, patients with AIP from the present study were jaundiced less severe and consequently were less likely to undergo pre-operative biliary drainage procedures, comparable to the report of Bledsoe [10]. (2): In contrast to the young age of both the AIP patients ( 34 and 49 years old) here, $80 \%$ of cases of PC occur in those over 60 years old, and rarely occurs before the age of $40[16,17]$. So, young age may help differentiate AIP from PC, especially for AIP type 2. (3): Tumor marker is a group of biomarkers found in blood that can be elevated by the presence of one or more types of cancer, each indicative of the presence of particular cancer. Tumor marker of CA19-9, CA 242 are frequently elevated in pancreatic cancer. However, with sensitivity of $80 \%$ and specificity of $73 \%$, they are used more for following known cases rather than diagnosis [18]. In the first case, tumor marker of CA50, CA19-9, CA 242, TPS and TPA was highly elevated, while in the second case, postoperative serum levels of tumor markers TPA and TPS were elevated (Table 1). Overall, both of AIP cases has elevated TPS and TPA, which rarely occurs in PC. The reason is unknown, but contrary to the utilization of serum tissue polypeptide specific antigen (TPS) and tissue polypeptide antigen (TPA) as complements to CA 199 in the detection of pancreatic carcinoma in the last 4 decades [19-21]. (4): Agreement concerning mass location, vascular involvement, pathological lymph nodes among morphologic imagings of US, CT, EUS, MRI, PET-CT were significantly higher in PC than in AIP [3]. That was also noted in the present report: for the first case, in contrast to mass in the pancreatic head by esophagogastroduodenoscopy, PET-CT and MRI, abdominal ultrasonography showed a tumor in the uncinate process of the pancreas while enhanced computed tomography (CT) revealed a mass in the end of lower bile duct. For the second case, abdominal CEUS demonstrated a malignant mass in the pancreatic head, whereas Helical CT and MRI scan with contrast enhancement, revealed a nodule in the end of the dilated lower bile in the enlarged pancreatic head. In all, for both of the cases, the result of imaging modality cannot recapitulated those seen in the other ones. That was different to $\mathrm{PC}$, whose similar observations by one imaging modality can usually be corroborated by other ones. The reason may be due to that the morphology of the mass and pancreas changes with the dynamic immune functional status [1113]. (5): no obesity and new-onset diabetes mellitus. There is congruent data to support the association between PC development and obesity, new-onset diabetes mellitus (DM). Large epidemiologic and cohort studies have identified obesity and new-onset DM as high-risk factors for early detection of PC [22, 23]. For AIP, however, only $21 \%$ of patients developed diabetes mellitus (pancreatic endocrine insufficiency), of whom 73\% required insulin [24]. In total, pancreatic endocrine insufficiency exist rather as long-term outcome than risk factor in patients with AIP, which may explain both of the AIP cases has no DM and obesity in the present study. (6): Comparable to this study, patients with AIP complained significantly more of pain as first presenting symptom, whereas more patients complained painless jaundice in the PC group $[1,10]$. In all, patients with AIP presented more often with pain and less often with jaundice. (7):Also, patients with AIP tended to smoke significantly more often $[3,10]$, contrary to the non-smoking habit of both cases from the present study. Research gaps and opportunities to address the interplay and underlying mechanisms between smoking and AIP, PC need be outlined in the future work.

\section{Conclusions}

In all, the clinical, laboratory and imaging presentation of patients with AIP and PC was very similar. Rather than relying on the rare typical signs, diagnosis of untypical AIP is probably best initiated by a panel of routine-check signs. We propose the combining absence or presence of above routine signs should make the clinician pay more attention to AIP diagnosis in PC-suspected patients in the decision-making process.

\section{Abbreviations}

AIP: autoimmune pancreatitis; IFL: indirect immunofluorescence; IgG4: immunoglobulin G4; ITM: immunoturbidimetry; PC: pancreatic cancer; WB: Western blotting

\section{Acknowledgements \\ The authors thank Chenshan Yuan (CY), Yubao Song, and Yang Zhong for helpful technical assistance.}

\section{Authors' contributions}

GL carried out the molecular genetic studies, participated in the sequence alignment and drafted the manuscript. WK, JZ participated in the design of the study and performed the statistical analysis. JM, ZG conceived of the study, and participated in its design and coordination and helped to draft the manuscript. All authors read and approved the final manuscript.

\section{Funding}

This work was supported by grants from Shanxi Province Science Foundation for Distinguished Young Scholar (2019), the doctor project of Shanxi Cancer Hospital, China (2017A06), National Natural Science Foundation of China for Young Scholars (Grant No: 81201810), science and research fund of Shanxi Health and Family Planning Commission (Grant No: 201601063), The Key research Project of Shanxi Province, China (socail development: 201703D321010-1), Natural Science Foundation of Guangdong Province, China (2015A030313057). The funders had no role in study design, data collection and analysis, decision to publish, or preparation of the manuscript.

\section{Availability of data and materials}

The datasets generated during and/or analysed during the current study are available from the corresponding author on reasonable request.

\section{Ethics approval and consent to participate}

This study have obtained consent to publish from human participants and were performed under conditions approved by the Ethics Committee of Shanxi cancer hospital (No. 201902), in compliance with the NIH Guidelines, items and Chinese relevant legislation for use of 
individual participant's data in any form (including images, videos, voice recordings etc).

\section{Consent for publication}

All the patient gave written consent for their personal or clinical details along with any identifying images to be published in this study and a copy of the patients' written consent is available for the journal.

\section{Competing interests}

The authors declare that they have no competing interests.

\section{Author details}

'Department of general surgery, Shanxi Cancer Hospital, Shanxi Medical University, Taiyuan, Shanxi Province, China. ${ }^{2}$ Department of critical care medicine, The first hospital of Shanxi medical University, The Hospital of Shanxi University of Traditional Chinese Medicine, Taiyuan, Shanxi Province, China. ${ }^{3}$ Department of general surgery, The Hospital of Shanxi University of Traditional Chinese Medicine, Taiyuan, Shanxi Province, China. ${ }^{4}$ Department of general surgery, Shanxi Dayi Hospital, Shanxi academy of Medical science, Taiyuan, Shanxi Province, China.

Received: 3 May 2019 Accepted: 3 October 2019

\section{Published online: 07 November 2019}

\section{References}

1. Matsumori T, Shiokawa M, Kodama Y. Pancreatic mass in a patient with an increased serum level of IgG4. Gastroenterology. 2018;155(2):269-70.

2. Bernasconi L, Mundwiler E, Regenass S, Aubert V, Hammerer-Lercher A, Heijnen I. Variable and inaccurate serum IgG4 levels resulting from lack of standardization in IgG subclass assay calibration. Clin Chem Lab Med. 2019.

3. Hsu WL, Chang SM, Wu PY, Chang CC. Localized autoimmune pancreatitis mimicking pancreatic cancer: case report and literature review. J Int Med Res. 2018;46(4):1657-65.

4. Otsuki M, Chung JB, Okazaki K, et al. Asian diagnostic criteria for autoimmune pancreatitis: consensus of the Japan-Korea symposium on autoimmune pancreatitis. J Gastroenterol. 2008;43(6):403-8.

5. Chari ST, Takahashi N, Levy MJ, et al. A diagnostic strategy to distinguish autoimmune pancreatitis from pancreatic cancer. Clin Gastroenterol Hepatol. 2009; 7(10):1097-103.

6. Khosroshahi A, Wallace ZS, Crowe JL, et al. International consensus guidance statement on the management and treatment of lgG4-related disease. Arthritis Rheum. 2015;67(7):1688-99.

7. de Castro SM, de Nes LC, Nio CY, et al. Incidence and characteristics of chronic and lymphoplasmacytic sclerosing pancreatitis in patients scheduled to undergo a pancreatoduodenectomy. HPB (Oxford). 2010; 12(1):15-21.

8. Adachi K, Hashimoto K, Nonaka R, et al. A case of an lgG4-related inflammatory Pseudotumor of the liver showing enlargement that was difficult to differentiate from hepatic Cancer. Gan To Kagaku Ryoho. 2017; 44(12):1922-4

9. Hamano H, Tanaka E, Ishizaka N, Kawa S. IgG4-related disease - a systemic disease that deserves attention regardless of One's subspecialty. Intern Med. 2018;57(9):1201-7.

10. Bledsoe JR, Della-Torre E, Rovati L, Deshpande V. IgG4-related disease: review of the histopathologic features, differential diagnosis, and therapeutic approach. APMIS. 2018;126(6):459-76.

11. Kubo S, Nakayamada S, Tanaka Y. Immunophenotype involved in IgG4related disease. Mod Rheumatol. 2018:1-13.

12. Hiratsuka I, Yamada H, Itoh M, et al. Changes in serum immunoglobulin G4 levels in patients with newly diagnosed Graves' disease. Exp Clin Endocrinol Diabetes. 2018.

13. Konno N, Sugimoto M, Takagi T, et al. Changes in N-glycans of lgG4 and its relationship with the existence of hypocomplementemia and individual organ involvement in patients with lgG4-related disease. PLoS One. 2018; 13(4):e0196163.

14. Bhattacharya A, Cruise M, Chahal P. Endoscopic ultrasound guided 22 gauge core needle biopsy for the diagnosis of autoimmune pancreatitis. Pancreatology. 2018;18(2):168-9.

15. Moon SH, Kim MH, Park DH, et al. Is a 2-week steroid trial after initial negative investigation for malignancy useful in differentiating autoimmune pancreatitis from pancreatic cancer? A prospective outcome study. Gut. 2008;57(12):1704-12

16. Danai LV, Babic A, Rosenthal MH, et al. Altered exocrine function can drive adipose wasting in early pancreatic cancer. Nature. 2018;558(7711):600-4.

17. Ryan DP, Hong TS, Bardeesy N. Pancreatic adenocarcinoma. N Engl J Med. 2014;371(11):1039-49.

18. Balachandran VP, Łuksza M, Zhao JN, et al. Identification of unique neoantigen qualities in long-term survivors of pancreatic cancer. Nature. 2017;551(7681):512-6.

19. Slesak B, Harlozinska-Szmyrka A, Knast W, Sedlaczek P, van Dalen A, Einarsson R. Tissue polypeptide specific antigen (TPS), a marker for differentiation between pancreatic carcinoma and chronic pancreatitis. A comparative study with CA 19-9. Cancer. 2000;89(1):83-8.

20. Talar-Wojnarowska R, Gasiorowska A, Olakowski M, Lekstan A, Lampe P, Malecka-Panas E. Clinical value of serum neopterin, tissue polypeptidespecific antigen and CA19-9 levels in differential diagnosis between pancreatic cancer and chronic pancreatitis. Pancreatology. 2010;10(6):689-94.

21. Basso D, Fabris C, Del FG, et al. Combined determination of serum CA 19-9 and tissue polypeptide antigen: why no improvement in pancreatic cancer diagnosis. Oncology. 1988;45(1):24-9.

22. Tirkes T, Shah ZK, Takahashi N, et al. Reporting standards for chronic pancreatitis by using $\mathrm{CT}, \mathrm{MRl}$, and MR Cholangiopancreatography: the consortium for the study of chronic pancreatitis, diabetes, and pancreatic Cancer. Radiology. 2019;290(1):207-15.

23. Tu J, Yang Y, Zhang J, et al. Effect of the disease severity on the risk of developing new-onset diabetes after acute pancreatitis. Medicine (Baltimore). 2018;97(22):e10713.

24. Vujasinovic M, Valente R, Maier P, et al. Diagnosis, treatment and long-term outcome of autoimmune pancreatitis in Sweden. Pancreatology. 2018; 18(8):900-4

\section{Publisher's Note}

Springer Nature remains neutral with regard to jurisdictional claims in published maps and institutional affiliations.

Ready to submit your research? Choose BMC and benefit from:

- fast, convenient online submission

- thorough peer review by experienced researchers in your field

- rapid publication on acceptance

- support for research data, including large and complex data types

- gold Open Access which fosters wider collaboration and increased citations

- maximum visibility for your research: over $100 \mathrm{M}$ website views per year

At BMC, research is always in progress.

Learn more biomedcentral.com/submissions 